\title{
Sintomatologia de depressão autorreferida por pacientes oncológicos em tempos de pandemia da COVID-19
}

Symptomatology of self-referred depression by oncological patients in times of the COVID-19 pandemic

Sintomatología de la depresión autoreferida por pacientes oncológicos en tiempos de pandemia COVID-19

Lydia Raquel Rodrigues Bastos ORCID: https://orcid.org/0000-0002-2540-8016 Faculdade Noroeste, Brasil E-mail: lydiaraque12015@gmail.com

Kelly Cristina Pereira dos Santos ORCID: https://orcid.org/0000-0002-4447-860X Faculdade Noroeste, Brasil E-mail: enfermeirakelly2@gmail.com

Marislene de Jesus Silva Ribeiro ORCID: https://orcid.org/0000-0002-2696-6247 Faculdade Noroeste, Brasil E-mail marislene.ribeiro@hotmail.com Caroline Christine Pincela da Costa ORCID: https://orcid.org/0000-0002-6732-913X Universidade Federal de Goiás, Brasil E-mail: carolinechristine@ hotmail.com

Leandro do Prado Assunção ORCID: https://orcid.org/0000-0002-1743-8151 Universidade Federal de Goiás, Brasil

E-mail: leandropadroassuncao@gmail.com Jessica da Silva Campos ORCID: https://orcid.org/0000-0002-6254-7250 Universidade Paulista, Brasil E-mail: jsilvacampos18@gmail.com

Júlio César Coelho do Nascimento ORCID: https://orcid.org/0000-0002-1783-842X Faculdade Noroeste, Brasil

E-mail: prof.julioccnascimento@gmail.com

\begin{abstract}
Resumo
Estudo transversal realizado com pacientes oncológicos com objetivo de estimar a prevalência de sintomas da depressão em tempos de pandemia da COVID-19. A coleta de dados foi realizada através da HADS (Hospital Anxiety and Depression Scale) 108 pacientes portadores de doenças oncológicas participaram deste estudo. Realizou-se uma análise descritiva e estatística para verificar a associação entre as variáveis. Utilizamos o teste qui-quadrado entre as variáveis com nível de significância de 0,05. Apenas as variáveis depressão e ansiedade, depressão e trabalho apresentaram estatística significativa com valor de $p$ menor que 0,05. A partir desses resultados foi feita uma regressão logística e o cálculo do odds ratio entre as variáveis 'depressão', 'trabalho' e 'ansiedade', no qual observa-se que pessoas que já trabalharam e não trabalham mais, tem uma maior chance (3,36 vezes) de apresentar um quadro de depressão. Além de tudo, pessoas com ansiedade também apresentam maiores chances (14,86 vezes) de depressão.
\end{abstract}

Palavras-chave: Depressão; Câncer; Pandemia por COVID-19.

\begin{abstract}
Cross-sectional study carried out with cancer patients with the aim of estimating the prevalence of symptoms of selfreported depression in times of the COVID-19 pandemic. Data collection was performed using the Hospital Anxiety and Depression Scale - HADS. 108 patients with cancer diseases participated in this study. A descriptive and statistical analysis was performed to verify the association between the variables. We used the chi-square test between the variables with a significance level of 0.05 . Thus, we noticed that only the variables depression and anxiety, depression and summary statistical work with p-value less than 0.05. Based on these results, a logistic regression was performed and the odds ratio was calculated between the variables depression, work and anxiety, in which we observed that people who have already worked and no longer work have a greater chance (3.36 times) of present a picture of depression. Furthermore, people with anxiety are also more likely (14.86 times) of depression.
\end{abstract}

Keywords: Depression; Cancer; COVID-19 Pandemic. 


\section{Resumen}

Estudio transversal realizado con pacientes oncológicos con el objetivo de estimar la prevalencia de síntomas de depresión autoinformada en tiempos de la pandemia COVID-19. La recolección de datos se realizó mediante la Escala Hospitalaria de Ansiedad y Depresión. En este estudio participaron 108 pacientes con enfermedades cancerosas. Se realizó un análisis descriptivo y estadístico para verificar la asociación entre las variables. Utilizamos la prueba de chi-cuadrado entre las variables con un nivel de significancia de 0.05. Así, notamos que solo las variables depresión y ansiedad, depresión y estadístico resumen funcionan con un valor de p menor a 0.05. A partir de estos resultados se realizó una regresión logística y se calculó la odds ratio entre las variables depresión, trabajo y ansiedad, en la que se observó que las personas que ya han trabajado y ya no trabajan tienen una mayor probabilidad (3,36 veces) de presentar un cuadro de depresión. Además, las personas con ansiedad también tienen más probabilidades $(14,86$ veces) de depresión.

Palabras clave: Depresión; Cáncer; Pandemia por COVID-19.

\section{Introdução}

O câncer é um dos principais problemas da saúde pública (Brasil, 2020). O tratamento é invasivo e é visto como um processo mutilador. Nesse sentido, é comum que pacientes enfrente desconfortos físicos e emocionais gerados pelas incertezas decorrentes do processo da doença. Conflitos emocionais, como depressão e medo podem associar-se a outros sofrimentos psicológicos, como ansiedade e estresse. Comumente, estes sentimentos se formam como medida de enfrentamento a doença (Miranda et al., 2015).

A depressão é um transtorno mental evidenciado por tristeza permanente correlacionada a sintomas de apatia, astenia, inapetência, mudanças no apetite, sonolência ou insônia, ansiedade, perda de concentração, indecisão, inquietude, sensação de impotência, culpa, desesperança e pensamentos suicidas ou de causar danos a si mesmos (World Health Organization, 2017).

Em pacientes oncológicos, a depressão influencia diretamente nos hábitos diários, e consequentemente reflete de forma negativa no processo de enfrentamento do câncer atingindo a qualidade de vida de forma mútua (Miranda et al., 2015).

A depressão atinge aproximadamente 300 milhões de pessoas no mundo. Destes, aproximadamente 5,8\% residem no Brasil (Brasil, 2017). Os sintomas podem se apresentar de forma leve, moderada e grave. Atualmente, dentre os fatores agravantes para as pessoas que vivem com a depressão, a pandemia da COVID-19, causada pelo vírus SARS-CoV-2 tem contribuído diretamente com os sinais de alarme no que refere à saúde mental (Brasil, 2020; Macedo Neto et al., 2020).

Considerando que o paciente com câncer apresenta sintomas depressivos durante o seu tratamento, acredita-se que este foi majoritariamente evidenciado pela pandemia por caracterizarem-se como indivíduos no grupo de risco. Por este motivo, torna-se crucial o conhecimento dos principais sintomas vivenciado pelos pacientes oncológicos neste cenário pandêmico.

Ao conhecê-los, acredita-se que será possível elaborar estratégias para promover qualidade de vida no período da pandemia. Diante disso, o presente estudo objetivou estimar a prevalência de sintomas da depressão autorreferida em pacientes em tratamento de câncer em tempos da pandemia da COVID-19.

\section{Metodologia}

Estudo descritivo-exploratório, transversal com os recursos da abordagem qualiquantitativo, aprovado pelo Comitê de Ética em Pesquisa com parecer de número: 4.577.751 CAAE: 43517221.1.0000.8011, conforme recomendação da Resolução 466/12 do Conselho Nacional de Saúde. A realização deste estudo contou com a utilização de ferramentas virtuais elaborados através do Google Forms aplicados entre o mês de abril e maio de 2021.

A população foi constituída por amostra de conveniência. Os pacientes incluídos neste estudo deveriam encontrar-se em tratamento de câncer por um período $\leq$ cinco anos e com sintomatologia de depressão autorreferida, idade $\leq 18$ anos e com 
a capacidade intelectual preservada para responder a Escala Hospitalar de Ansiedade e Depressão (Hospital Anxiety and Depression Scale - HADS), bem como o questionário socioeconômico.

Foram excluídos pacientes que não responderam o questionário completamente, bem como aqueles que afirmaram não apresentar nenhum sintoma de depressão durante o período da pandemia da COVID-19.

Os participantes foram convidados a participar da pesquisa por meio eletrônico (WhatsApp ${ }^{\oplus}$, Telegram $^{\oplus}$, Facebook $^{\oplus}$, Instagram $^{\odot}$. Após o aceite e assinatura do Termo de Consentimento Livre e Esclarecido (TCLE), encaminhou-se o link do questionário para que este fosse respondido.

Realizou-se uma análise descritiva com todas as variáveis, em que foram colocadas a quantidade de cada subclasse e a porcentagem. Posteriormente, apresenta-se os gráficos das variáveis sociodemográficas (Idade, sexo, Raça, Estado Civil, Escolaridade e Renda). Dessa mesma forma também foram feitas análise descritiva com tabelas e gráficos dos dados clínicos que foram o tempo de diagnóstico, tratamento submetido, tipo de câncer, COVID19, Depressão e Ansiedade.

Após as análises descritivas foram feitos os testes estatísticos para verificar a associação entre as variáveis. Utilizou-se o teste qui-quadrado entre todas as variáveis com nível de significância de 0,05 . Todas as análises foram feitas com auxílio de planilha eletrônicas para montagem das tabelas e com software R para realização dos testes estatísticos.

\section{Resultados e Discussão}

Participaram deste estudo 108 pacientes moradores de diferentes regiões brasileiras, ganhando destaque no estado de Goiás, com 35 pacientes, e São Paulo, com 32 pacientes. A Tabela 1 descreve as características sociodemográficas dos entrevistados. A idade dos participantes concentrou indivíduos entre a faixa etária de 30 a 40 anos (31,5\%), seguido das faixas entre 40 e $50(25,9 \%)$ anos e 50 e 60 anos $(16,7 \%)$.

Tabela 1 - Características sociodemográficas dos entrevistados. Goiânia-GO, 2021.

\begin{tabular}{|c|c|c|}
\hline Variáveis/Estatística & $\mathbf{n}$ & $\%$ \\
\hline \multicolumn{3}{|l|}{ Idade } \\
\hline Entre 18 e 20 anos & 2 & $1,9 \%$ \\
\hline Entre 20 e 30 anos & 14 & $13,0 \%$ \\
\hline Entre 30 e 40 anos & 34 & $31,5 \%$ \\
\hline Entre 40 e 50 anos & 28 & $25,9 \%$ \\
\hline Entre 50 e 60 anos & 18 & $16,7 \%$ \\
\hline Entre 60 e 70 anos & 8 & $7,4 \%$ \\
\hline Acima de 70 anos & 4 & $3,7 \%$ \\
\hline \multicolumn{3}{|l|}{ Sexo } \\
\hline Feminino & 97 & $89,8 \%$ \\
\hline Masculino & 11 & $10,2 \%$ \\
\hline \multicolumn{3}{|l|}{ Estado Civil } \\
\hline Solteiro (a) & 21 & $19,4 \%$ \\
\hline Casado (a) & 65 & $60,2 \%$ \\
\hline Divorciado (a) & 11 & $10,2 \%$ \\
\hline Viúvo (a) & 4 & $3,7 \%$ \\
\hline Outros & 7 & $6,5 \%$ \\
\hline
\end{tabular}

Escolaridade 


$\begin{array}{lll}\text { Ensino Médio } & 35 & 32,4 \% \\ \text { Ensino Superior } & 37 & 34,3 \% \\ \text { Pós-graduação } & 24 & 22,2 \%\end{array}$

\section{Etnia}

\begin{tabular}{|c|c|c|}
\hline Branca & 107 & $99,1 \%$ \\
\hline Parda & 1 & $0,9 \%$ \\
\hline \multicolumn{3}{|l|}{ Renda Familiar } \\
\hline Até 1 salário mínimo & 22 & $20,4 \%$ \\
\hline 1 a 2 salários mínimos & 28 & $25,9 \%$ \\
\hline 2 a 4 salários mínimos & 22 & $20,4 \%$ \\
\hline Mais de 4 salários mínimos & 36 & $33,3 \%$ \\
\hline \multicolumn{3}{|l|}{ Com quem vive } \\
\hline Sozinho (a) & 8 & $7,4 \%$ \\
\hline Cônjuge & 53 & $49,1 \%$ \\
\hline Companheiro (a) & 10 & $9,3 \%$ \\
\hline Filho (a) & 23 & $21,3 \%$ \\
\hline Neto (a) & 1 & $0,9 \%$ \\
\hline Outras pessoas & 13 & $12,0 \%$ \\
\hline \multicolumn{3}{|l|}{ Atividade ocupacional } \\
\hline Nunca trabalhou & 2 & $1,9 \%$ \\
\hline Já trabalhou, mas não trabalha mais & 36 & $33,3 \%$ \\
\hline Trabalha, mas não em atividade atualmente & 13 & $12,0 \%$ \\
\hline Trabalha em atividade atualmente & 57 & $52,8 \%$ \\
\hline \multicolumn{3}{|l|}{ Razão de estar sem atividade } \\
\hline Afastado por doença & 14 & $13,0 \%$ \\
\hline Aposentado por doença/invalidez & 7 & $6,5 \%$ \\
\hline Aposentado por tempo de trabalho/idade & 11 & $10,2 \%$ \\
\hline Dona de casa/cuida de casa/Afazeres domésticos & 15 & $13,9 \%$ \\
\hline Está procurando, mas não consegue trabalho & 6 & $5,6 \%$ \\
\hline Estudos e treinamento & 4 & $3,7 \%$ \\
\hline Sem resposta & 51 & $47,2 \%$ \\
\hline
\end{tabular}

Fonte: Autores.

A maior parte dos pacientes era do sexo feminino (89,8\%) e casado $(60,2 \%)$. Nesse sentido, $49,1 \%$ dos entrevistados relataram dividir a residência com seu cônjuge, 21,3\% com filho (a), e 9,3\% com seus companheiros.

Para a variável "escolaridade", observa-se índices estatisticamente próximos para os subgrupos 'Ensino Médio' e 'Ensino Superior', com 32,4\% e 34,3\%, respectivamente. Para a variável 'renda familiar', o estrato com mais de 4 salários mínimos obteve destaque, com representação de 33,3\% dos entrevistados, seguido de entre um e dois salários mínimos $(25,9 \%)$.

A variável 'atividade ocupacional' apontou que 52,8\% encontravam em exercício, seguido de pacientes que trabalharam em algum momento da vida, mas não estava mais em atividade (33,3\%). Indivíduos que trabalharam, mas não estavam em atividade durante a pesquisa, ou que nunca trabalharam, representaram $12,0 \%$ e $1,9 \%$ respectivamente. 
A principal razão de estarem sem atividades ocupacionais não foi caracterizada (47,2\%). Entretanto, 13,9\% dos entrevistados responderam que não estão trabalhando por envolvimento com atividades domésticas, 13,0\% estavam afastados do trabalho por doença, e 10,2\% eram aposentados por tempo de trabalho ou idade.

Dentre os aspectos clínicos, observou-se que a maior parte dos pacientes haviam sido diagnosticados com câncer entre um e dois anos $(36,1 \%$ e $35,2 \%)$ do período de entrevista. Além disso, o principal tratamento ao qual esses pacientes foram/estavam sendo submetidos foi a cirurgia (50,0\%), seguido de cirurgia somada a quimioterapia (14,8\%), e cirurgia somada a quimioterapia e radioterapia $(8,3 \%)$.

Para a variável 'tipo de câncer', observa-se uma ampla distribuição quanto ao sítio anatômico acometido pela neoplasia. Entretanto, o câncer de tireoide foi o mais prevalente, com 38,0\%. Outras variantes histopatológicas para o câncer de tireoide também se destacaram, como o carcinoma papilífero de tireoide $(6,5 \%)$ e tireoide-papilífero $(3,7 \%)$. O câncer de mama também obteve destaque, sendo citado por 15,7\% das entrevistadas. Outros tipos de câncer relatados com frequência no estudo foram: intestino, próstata, pulmão, com 3,7\% cada, e ovário, com 1,9\%.

Dentre os entrevistados, 13,9\% afirmaram ter contato com o vírus SARS-CoV-2, desenvolvendo a COVID-19. A distribuição entre essa infecção, bem como de sintomas de ansiedade e depressão estão descritas na Tabela 2. Muitos pacientes relataram a possibilidade/probabilidade de estarem com sintomas de ansiedade, representando 20,4\% e 45,4\%, respectivamente.

Tabela 2 - Distribuição de infectados pela COVID-19 e dos sintomas de ansiedade e depressão entre os entrevistados. Goiânia-GO, 2021.

\begin{tabular}{llc}
\hline Variáveis/Estatística & $\mathbf{n}$ & \% \\
\hline COVID-19 & & $13,9 \%$ \\
\hline Sim & 15 & $86,1 \%$ \\
Não & 93 & \\
\hline Ansiedade & 37 & $34,3 \%$ \\
\hline Improvável & 22 & $20,4 \%$ \\
Possível & 49 & $45,4 \%$ \\
Provável & & \\
\hline Depressão & 47 & $43,5 \%$ \\
\hline Improvável & 37 & $34,3 \%$ \\
Possível & $24,2 \%$ \\
Provável & 24 & $22 \%$ \\
\hline
\end{tabular}

Fonte: Autores.

Para sintomas de depressão, observou-se que 43,5\% dos pacientes afirmaram que é improvável estarem apresentando sintomatologias depressivas. No entanto, a somatória de pacientes para os estratos possível e provável quanto aos sintomas de depressão ultrapassam a metade dos entrevistados (56,5\%), sendo 34,3\% para possivelmente com sintomas depressivos, e $22,2 \%$ para provavelmente com sintomas depressivos.

A associação entre as variáveis sociodemográficas com os sintomas de ansiedade e depressão estão descritos na Tabela 3. A variável atividade ocupacional mostrou associação significativa com a depressão ( $\mathrm{p}=0,001)$. Os sintomas de ansiedade e depressão também foram fortemente associáveis entre si ( $\mathrm{p}=0,000003)$. 
Tabela 3 - Relação entre as variáveis sociodemográficas com os sintomas de ansiedade e depressão. Goiânia-GO, 2021.

\begin{tabular}{llll}
\hline Variáveis $^{\mathbf{a}}$ & COVID-19 $^{\mathbf{b}}$ & Ansiedade $^{\mathbf{b}}$ & Depressão $^{\mathbf{b}}$ \\
\hline Idade & 0,78 & 0,55 & 0,24 \\
Sexo & 0,37 & 0,62 & 0,64 \\
Estado Civil & 0,82 & 0,94 & 0,56 \\
Escolaridade & 0,6 & 0,12 & 0,17 \\
Etnia & 0,29 & 0,99 & 0,89 \\
Renda familiar & 0,9 & 0,09 & 0,3 \\
Com quem reside & 0,18 & 0,41 & 0,64 \\
Atividade Ocupacional & 0,08 & 0,11 & 0,001 \\
COVID-19 & 1 & 0,71 & 0,51 \\
Ansiedade & 0,39 & 1 & 0,000003 \\
Depressão & 0,51 & 0,0000003 & 1 \\
\hline
\end{tabular}

$\mathrm{a}$ - Teste de associação qui-quadrado $\left(\chi^{2}\right) ; \mathrm{b}$ - p-valor. Fonte: Autores.

A regressão logística da varável depressão com ansiedade e trabalho, demonstrada na Tabela 4, revelou que não estar mais trabalhando pode ser um fator de risco para o desenvolvimento de um quadro depressivo $(\mathrm{OR}=3,36 ; \mathrm{IC} 95 \%=1,17-11,19$; $\mathrm{p}=0,03$ ). Adicionalmente, a presença de sintomas depressivos também foi fortemente associada a sintomas de ansiedade, aumentando em até 14 vezes o risco de desenvolvimento desse quadro (IC95\%=5,03-53,72; p=0,000005).

Tabela 4 - Relação entre as variáveis sociodemográficas com os sintomas de ansiedade e depressão. Goiânia-GO, 2021.

\begin{tabular}{llll}
\hline Variável $^{\mathbf{a}}$ & Estimador & p-valor & Odds Ratio (IC. 95\%) \\
\hline Trabalho & & & \\
\hline Trabalha em atividade atualmente & & - & - \\
Já trabalhou, mas não trabalha mais & 1,21 & 0,03 & $3,36(1,17-11,19)$ \\
Nunca trabalhou & 19,81 & 0,99 & $4,03(0-$ Inf $)$ \\
Trabalha, mas não em atividade atualmente & 18,71 & 0,99 & $4,49(0-$ Inf $)$ \\
\hline Ansiedade & & & - \\
Não & Ref & - & $14,86(5,03-53,72)$ \\
Sim & 2,69 & 0,000005 & \\
\hline
\end{tabular}

a - Regressão logística. Fonte: Autores.

Considerando as características sociodemográficas, os resultados do estudo são semelhantes a outros realizados em indivíduos diagnosticados com alguma neoplasia, mostrando maior frequência de pacientes do sexo feminino, casadas, e com idade entre 30 e 40 anos (Andrade, Sawada \& Barichello, 2013; Kawakami et al., 2014).

O sexo feminino foi o mais prevalente entre os pacientes entrevistados, corroborando com dados levantados pela literatura. Evidências têm apontado o sexo feminino com um dos mais afetados por sintomas de ansiedade e depressão durante a pandemia por COVID-19. Estudos relataram maior prevalência de alterações psicossociais em mulheres durante esse período (Barros et al., 2020; Ozamiz-Etxebarria et al., 2020).

Estudos realizados com a população brasileira conduzidos previamente a pandemia também relatavam maiores queixas de saúde e transtornos mentais oriundos de mulheres (Gonçalves et al, 2018; Munhoz et al., 2018). Nesse sentido, o diagnóstico de um câncer somado ao contexto pandêmico atualmente vivenciado, podem exacerbar esses sintomas. A constatação de um índice maior de entrevistados de sexo feminino pode refletir a maior atenção das mulheres com sua saúde, 
também apontando maior cuidado com sinais e sintomas referentes a alterações da saúde mental. Apesar disso, não encontrouse relação estatisticamente significativa entre a variável 'sexo e os sintomas de ansiedade e depressão'.

A maior parte dos diagnósticos de câncer foi registrada entre os últimos dois anos, período que converge ao da pandemia, alguns fatores intrínsecos ao risco para COVID-19 podem ter agravado esses sintomas. A necessidade do distanciamento social e consequentemente, maior frequência do tempo dentro de casa, com privação da interação social podem recair sobre a intensificação dessas alterações psicossociais. Além disso, a sensação de medo por pertencer ao grupo de risco e ausência de certeza sobre o que pode decorrer de uma infecção pelo vírus SARS-CoV-2, podem não somente acentuar a sintomatologia de ansiedade e depressão, mas também levar ao desenvolvimento de fobias sociais e síndrome do pânico (Miranda et al., 2015).

Quanto à idade dos entrevistados, a faixa etária com mais participantes foi de entre 30 e 40 anos $(31,5 \%)$. O acometimento por neoplasias caracteriza-se como uma comorbidade entre esses pacientes, e assim, define tais indivíduos como grupo de risco para a infecção por SARS-CoV-2. Em estudo elencou nove fatores de risco associados à ansiedade ou sintomas depressivos: idade mais jovem, renda reduzida, câncer ou outras doenças crônicas, membros da família vivendo com câncer, preocupações relacionadas à infecção por COVID-19 para si ou para membros da família, morar sozinho, ter conflitos familiares, ter $<3$ ou $>8$ horas de sedentarismo por dia e piora da qualidade do sono (Guo et al., 2020). Evidenciado em estudo, realizado com a população brasileira, também apresentou maior frequência de indivíduos mais jovens com tais quadros psicossociais (Barros et al., 2020; Guo, et al., 2020).

Neste estudo, evidenciou-se a relação entre indivíduos mais jovens e o diagnóstico com câncer. Apesar de não avaliado os entrevistados, especialmente os que não tiveram COVID-19 - a maioria neste estudo -, sobre o medo de contrair a doença, supõe que encontrar-se sob uma população de alto risco para a doença certamente influenciou o aumento nos sentimentos de ansiedade e depressão, o que pode ser confirmado pela alta percentagem de indivíduos que afirmaram a possibilidade/probabilidade de estarem apresentando algum quadro sintomatológico para esses transtornos. Todavia, não houve correlação estatística para as variáveis COVID-19 e idade com a presença desses transtornos mentais.

Com relação aos tipos de câncer, o sítio anatômico frequentemente acometido foi a tireoide. Segundo o Instituto Nacional de Câncer (INCA), os cânceres que afetam a glândula tireoide ocupam o quinto lugar entre os mais frequentes em mulheres, justificando assim a alta prevalência desse câncer na amostra. Ademais, um dos tratamentos mais empregados para tumores de tireoide é a cirurgia, o que é corroborado pelos nossos dados (Instituto Nacional de Câncer, 2021).

Os resultados desse estudo também encontraram uma associação entre a atividade ocupacional com a existência de sintomas depressivos $(\mathrm{p}=0,001)$. Além disso, os sintomas de ansiedade e a depressão foram associados entre si ( $\mathrm{p}=0,00003)$. Para a variável atividade ocupacional, mais da metade dos entrevistados $(52,8 \%)$ afirmaram trabalhar e estar em atividade atualmente.

Correlacionando os sintomas psicossociais analisados com essa variável, o aumento da frequência de pacientes com transtornos mentais pode estar relacionado com a mudança brusca de convivência e socialização. Uma vez que o distanciamento social aplicado, reduz-se a interação entre pessoas. Além disso, o modelo de trabalho presencial foi recorrentemente substituído pelo modelo remoto ("home office”) (Király et al., 2020).

Todavia, a análise de regressão logística da variável depressão com as variáveis trabalho e ansiedade revelaram associação entre o estrato 'Já trabalhou, mas não trabalha mais' e a presença de sintomas depressivos, mostrando 3,36 chances a mais de desenvolver a transtorno $(\mathrm{p}=0,03)$. Apesar disso, o principal motivo de os entrevistados não estarem exercendo alguma atividade profissional atualmente não foi caracterizado (47,2\%). Adicionalmente, a presença de sintomas de ansiedade também foi fortemente associada a presença de quadros depressivos, demonstrando que pacientes com ansiedade possuem 14,86 chances de desenvolver depressão $(\mathrm{p}=0,000005)$. 
É importante salientar que a própria condição de paciente com neoplasia pode levar ao surgimento de sintomas de ansiedade e depressão. A presença desses transtornos mentais é potencialmente danosa a esses pacientes e prejudica seu estado de saúde geral e qualidade de vida (Santos, Mota \& Pimenta, 2009). O acometimento pela própria doença oncológica pode ter desencadeado esse quadro sintomatológico de ansiedade e depressão, potencialmente exacerbado pela pandemia por COVID19.

No entanto, é inegável a amplitude da situação mental de pacientes, especialmente dentre o grupo de risco, durante o período pandêmico decorrente da COVID-19, destacando a necessidade de cuidados voltados para a saúde mental. A necessidade de privação de um amplo convívio social implica em forte impacto sobre a saúde mental da população.

O enfretamento de duas doenças que ilustram momentos difíceis requer o acompanhamento de profissionais habilitados para o manejo dessas condições. Nesse sentido, a Organização Mundial de Saúde (OMS), disponibiliza e divulga guias com medidas para preservação da saúde mental, destacando a urgência do suporte psicológico durante o período de pandemia (World Health Organization, 2020). Assim, conhecer e entender os sentimentos desse segmento da população permite o planejamento de ações que propiciem o bem-estar físico e mental dos indivíduos, fornecendo uma melhor qualidade na prestação de assistência à saúde.

\section{Considerações Finais}

Esse estudo objetivou estimar a prevalência de sintomas da depressão autorreferida em pacientes em tratamento de câncer em tempos de pandemia da COVID-19. Os resultados encontraram uma associação entre a atividade ocupacional com a existência de sintomas depressivos. A regressão logística e o cálculo do odds ratio permitiu observar que as pessoas que não estavam trabalhando durante o período da pandemia tiveram maior chance de apresentar quadro de pressão.

A amostra por conveniência dificultou uma representação homogênea da população. Outros aspectos relacionados com a condução da pesquisa também devem ser considerados, como a totalidade das alternativas fornecidas para resposta do entrevistado e a duração da pesquisa, que necessitou perdurou por um período relativamente pequeno, apenas um mês. Adicionalmente, o questionário não foi respondido por indivíduos de todas as regiões brasileira. Todavia, apesar desses possíveis vieses de seleção e interpretação de resultados, esses podem ser considerados representativos e condizentes com a literatura.

\section{Referências}

Andrade V., Sawada, N. O., Barichello, E. Qualidade de vida de pacientes com câncer hematológico em tratamento quimioterápico. Revista da Escola de Enfermagem da USP 2013; 47(2): 355-61.

Barros, M. B. A., Lima, M. G., Malta, D. C., Szwarcwald, C. L., Azevedo, R. C.S. D., Romero, D., et al. Relato de tristeza/depressão, nervosismo/ansiedade e problemas de sono na população adulta brasileira durante a pandemia de COVID-19. Epidemiologia e Serviços de Saúde $2020 ; 29$ (4): e2020427.

Brasil. Organização Pan-Americana da Saúde/Organização Mundial da Saúde. Depressão: o que você precisa saber. 2017.

Brasil. Ministério da Saúde. Instituto Nacional de Câncer. 2020 . https://www.inca.gov.br/sites/ufu.sti.inca.local/files//media/document//estimativa-2020-incidencia-de-cancer-no-brasil.pdf

Gonçalves, A. M. C., Gamarra, C. J., Lopes, C. S., Silva, G. A., Gama, J. R. A., Duque, K. C. D., et al. Prevalência de depressão e fatores associados em mulheres atendidas pela Estratégia de Saúde da Família. Jornal Brasileiro de Psicologia. 2018; 67(2): 101-9.

Guo, Y., Cheng, C., Zeng, Y, Li, Y., Zhu, M., Yang, W., et al. Mental health disorders and associated risk factors in Quarantined adults during the COVID-19 outbreak in China: cross-sectional study. Journal of medical Internet research. 2020; 22(8): e20328.

Instituto Nacional de Câncer - INCA. Estatísticas de Câncer. https://www.inca.gov.br/numeros-de-cancer..

Kawakami, D. M., Olah, S. L., Pivetta, N. R. S., Silva, R. C. R., Santos, D. C. N., Napoleão, L. L., et al. Avaliação da ansiedade e depressão de pacientes oncológicos que realizam quimioterapia ambulatorial. Colloq Vitae 2014; 6(3): 35-41.

Király, O., Potenza, M. N., Stein, D. J., King, D. L., Hodgins, D. C., Saunders, J. B., et al. Preventing problematic internet use during the COVID-19 pandemic: Consensus guidance. Comprehensive Psychiatry. 2020; 100: 152180. 
Research, Society and Development, v. 10, n. 13, e65101321058, 2021

(CC BY 4.0) | ISSN 2525-3409 | DOI: http://dx.doi.org/10.33448/rsd-v10i13.21058

Miranda, S. L. D., Lanna, M. A. L., Felippe, W. C. Espiritualidade, Depressão e Qualidade de Vida no Enfrentamento do Câncer: Estudo Exploratório. Psicologia: Ciência e Profissão [online]. 2015, v. 35, n. 3, pp. 870-885.

Munhoz, T. N., Nunes, B. P., Wehrmeister, F. C., Santos, I. S., Matijasevich, A. A nationwide population-based study of depression in Brazil. J Affect Disord. 2016;192:226-233.

Macedo Neto, A. O., Souza, B. C., Torres, F. C., Lima, H. R. F. S., Martins, L. P. S., Vaz-Tostes, L. P., et al. Desenvolvimento científico na área de saúde mental. In: Lourenço TTGMV. Livro de resumos: XVII Jornada Acadêmica de Saúde Mental da Faculdade de Medicina da Universidade Federal de Minas Gerais. 1 ed. Belo Horizonte: UFMG; 2020.P. 8-10.

Ozamiz-Etxebarria N, Dosil-Santamaria M, Picaza-Gorrochategui M, Idoiaga-Mondragon N. Niveles de estrés, ansiedad y depresión en la primera fase del brote del COVID-19 en una muestra recogida en el norte de España. Cad Saúde Pública. 2020; 36(4): e00054.

Santos, J., Mota, D. D. C. F., Pimenta, C. A. M. Co-morbidade fadiga e depressão em pacientes com câncer colo-retal. Revista da Escola de Enfermagem da USP 2009; 43 (4): 909-914. Disponível em: https://doi.org/10.1590/S0080-62342009000400024 .

World Health Organization - WHO. Depression and Other Common Mental Disordes: Global Health Estimates. World Health Organization ed. Geneva2017.

World Health Organization - WHO. Mental health and psychosocial considerations during the COVID-19 outbreak. 2020. Vhttps://www.who.int/docs/defaultsource/coronaviruse/mental-health-considerations.pdf?sfvrsn=6d3578af_2. 